\title{
An unusual form of TAC-TAC sine autonomic phenomena
}

\author{
Neil Lewis Pugach
}

Received: 21 June 2008/Accepted: 15 July 2008/Published online: 7 August 2008

(C) Springer-Verlag 2008

\begin{abstract}
The trigeminal autonomic cephalalgias (TACs) are characterized by the cardinal combination of shortlasting unilateral pain and autonomic phenomena affecting the head, including cluster headache, paroxysmal hemicrania and SUNCT. Infrequently reported have been cases with dissociation of these two cardinal features- usually with autonomic phenomena without pain, rather than the reverse. Herein is described the case of a 56-year-old man presenting with an unusual form of TAC resembling chronic paroxysmal hemicrania but with a lower frequency of occurrence, with temporal features not typical of previously described TACs, and consisting of severe unilateral head pain without any autonomic symptoms or signs, absolutely responsive to indomethacin
\end{abstract}

Keywords Trigeminal autonomic cephalalgia .

Paroxysmal hemicrania $\cdot$ Dissociation .

Trigeminal-autonomic reflex

\section{Introduction}

The trigeminal autonomic cephalalgias (TACs) are a group of headache disorders characterized by the cardinal combination of short-lasting pain and autonomic phenomena affecting the head. These include cluster headache $(\mathrm{CH})$, paroxysmal hemicrania and SUNCT. There is considerable overlap in the features of these entities, with individual defining characteristics predominantly relating to temporal factors and response or lack thereof to indomethacin. [1-3]

N. L. Pugach $(\bowtie)$

Headache Institute of Tidewater, Brighton Research Group,

Virginia Beach, VA, USA

e-mail: npugach@brg.cc
Rarely there have been reports of dissociation of the pain and autonomic symptoms/signs-most such reports describe autonomic symptoms/signs without the pain, and not the reverse [4-6]. Herein is described a case of pain without autonomic phenomena, with temporal features that are not entirely typical of any of the heretofore described TACs. To the author's knowledge, this is the first such case reported.

\section{Case report}

A 56-year-old male was initially seen on 5th February 2007. When he was in his mid-20s he began having headache episodes. He would have only a single occasional such episode until 2002 when they increased in frequency to a few times a week, then around 2004 they increased to daily, generally once a day, occasionally twice a day, and rarely 3 times a day, but never more than 3 times a day. When they were only occasional they would last up to $7 \mathrm{~h}$. However, when the frequency increased, they changed in duration to 20-30 min, occurring at any time of day, only rarely awakening him from sleep, with $60 \%$ occurring in mid-morning. The pain was described as "like a screwdriver twisting" invariably in the left orbit or temporal region, 8/10 in severity, with no associated aura, nausea, vomiting, photophobia, phonophobia, rhinorrhea, forehead or other facial sweating, nasal congestion, eyelid ptosis or edema, anisocoria, lacrimation or conjunctival injection. He would sit down and rub his eye during an episode (this would not help). He was unaware of any triggers, including $\mathrm{EtOH}$, napping, neck movements or manual pressure on the neck. He had never been prescribed any medication or oxygen for these, having taken only OTC's, which did not help. He had had a CT scan of the head 2 years previously, 
which he reported as having been unrevealing. He knew of no relevant family hx. Past medical history was remarkable only for controlled hypertension, and hyperlipidemia. He is a non-smoker and non-drinker. General and neurological exams were entirely unremarkable.

At the initial visit he was started on indomethacin 25 tid with instructions to increase it if necessary. He returned on 26th February 2007, indicating that since the second day of taking indomethacin 25 tid he had not had a single episode, and he had continued on this dose, but wanted to switch to the slow release formulation. He was switched to $75 \mathrm{mg} /$ day of the slow release. He returned on 24th October 2007, indicating that a $75 \mathrm{mg}$ dose taken every $36 \mathrm{~h}$ essentially completely prevented the episodes. Upon telephone followup on 27th December 2007, this same dosing schedule of indomethacin was continuing to completely control the episodes. During this conversation, he denied any ear pain or redness associated with the episodes, and also denied any associated unusual, non-pain symptoms whatsoever.

\section{Discussion}

This case is atypical in a number of aspects, most notably the complete absence of any autonomic symptoms or signs, therefore not meeting criterion $\mathrm{C}$ of the ICHD-II definition of paroxysmal hemicrania, which it most closely resembles. The temporal pattern, occurring in single episodes lasting up to $7 \mathrm{~h}$ each, only occasionally, for many years before increasing in frequency is atypical as well. The condition does meet criteria A, B, E and F, namely, regarding the total number of attacks, severity and location of pain (including unilaterality), duration of attacks, complete prevention of attacks by indomethacin and lack of other cause. However, with a maximum of 3 episodes per day (and this occurring only rarely, with the usual frequency being 1 episode per day), the condition does not meet criterion D [3]. Nor does it meet criteria for the other two TACs, CH or SUNCT.

Boes et al. [7] reported a case of chronic paroxysmal hemicrania that presented with pain location restricted to one ear, and no typical autonomic symptoms or signs. However, this patient did experience associated ipsilateral ear redness and probable edema, therefore, she did have autonomic signs, albeit atypical ones. Pareja [4] reported "partial attacks" of chronic paroxysmal hemicrania with autonomic phenomena but mild or no pain-however, this dissociation is the opposite of our patient's presentationfurthermore this dissociation of symptoms occurred in his patient only after a course of indomethacin, possibly as a withdrawal type of phenomenon. Bingel and Weiller [8] reported a case of indomethacin-responsive paroxysmal hemicrania without autonomic phenomena, with typical temporal characteristics. However, this was episodic and not chronic paroxysmal hemicrania, and also presented with bilateral rather than unilateral pain.

Ekbom [9] and Nappi [10] each reported a small percentage of their cluster headache populations having no autonomic symptoms-however, these patients were apparently otherwise typical cluster cases.

Assuming that the autonomic phenomena associated with the TACs are a result of the trigeminal-autonomic reflex, one might surmise the existence of a functional or anatomical disruption of this putative pathway in this patient. A literature search turned up no reports in this regard-perhaps functional brain imaging will ultimately clarify the nature of cases like the abovementioned.

In conclusion, a patient is described who demonstrates a syndrome with all the features of typical chronic paroxysmal hemicrania except for the frequency of episodes (typically 1/day, maximum 3/day) and with no associated autonomic phenomena whatsoever.

Acknowledgments The author thanks Dr. Fred Sheftell for his thoughtful comments.

Conflict of interest None.

\section{References}

1. Goadsby PJ (2001) Trigeminal Autonomic Cephalalgias (TACs). Acta Neurol Belg 101:10-19

2. Goadsby PJ, Lipton RB (1997) A review of paroxysmal hemicranias, SUNCT syndrome and other short-lasting headaches with autonomic feature, including new cases. Brain 120:193-209

3. Headache Classification Subcommittee of the International Headache Society. Classification and diagnostic criteria for headache disorders, cranial neuralgias and facial pain (2004) Cephalalgia 24(Suppl. 1):44-49

4. Pareja JA (1995) Chronic paroxysmal hemicrania: dissociation of the pain and autonomic features. Headache 35:111-113

5. Leone M, Rigamonti A, Bussone G (2002) Cluster headache sine headache: two new cases in one family. Cephalalgia 22(1):12-14

6. Salvesen R (2000) Cluster headache sine headache: case report. Neurology 55(3):451

7. Boes CJ, Swanson JW, Dodick DW (1998) Chronic paroxysmal hemicrania presenting as otalgia with a sensation of external acoustic meatus obstruction: two cases and a pathophysiologic hypothesis. Headache 38:787-791

8. Bingel U, Weiller C (2005) An unusual indomethacin-sensitive headache: a case of bilateral episodic paroxysmal hemicrania without autonomic symptoms? Cephalalgia 25(2):148-150

9. Ekbom K (1990) Evaluation of clinical criteria for cluster headache with special reference to the classification of the International Headache Society. Cephalalgia 10:195-197

10. Nappi G, Micieli G, Cavallini A et al (1992) Accompanying symptoms of cluster attacks: their relevance to the diagnostic criteria. Cephalalgia 3:165-168 